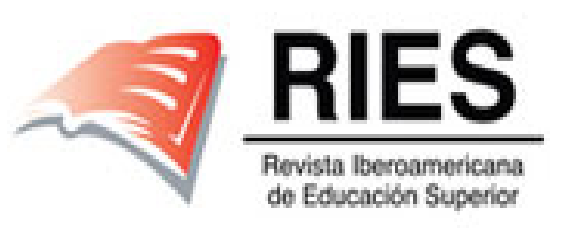

Revista Iberoamericana de Educación Superior

E-ISSN: 2007-2872

emmaro@unam.mx

Instituto de Investigaciones sobre la

Universidad y la Educación

México

Lavín-Verástegui, Jesús; Farías-Martínez, Gabriela-María

Perfil y prácticas educativas del docente orientado a la innovación en las escuelas de negocios en México

Revista Iberoamericana de Educación Superior, vol. III, núm. 6, enero-abril, 2012, pp. 117-127

Instituto de Investigaciones sobre la Universidad y la Educación

.jpg, México

Disponible en: http://www.redalyc.org/articulo.oa?id=299129030006

Cómo citar el artículo

- Número completo

- Más información del artículo

Página de la revista en redalyc.org

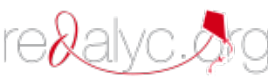

Sistema de Información Científica

Red de Revistas Científicas de América Latina, el Caribe, España y Portugal

Proyecto académico sin fines de lucro, desarrollado bajo la iniciativa de acceso abierto 


\title{
Perfil y prácticas educativas del docente orientado a la innovación en las escuelas de negocios en México
}

Jesús Lavín-Verástegui y Gabriela-María Farías-Martínez

\begin{abstract}
Resumen
Es de especial trascendencia para las escuelas de negocios en México afrontar las demandas sociales y de calidad académica de la educación superior. Ante el desafío del cambio, el profesor es el actor central en los procesos de innovación educativa. El objetivo principal de este trabajo de investigación exploratorio-descriptivo, fue conocer las características del perfil y de las prácticas educativas de un profesor innovador en las escuelas de negocios en México. Para lograr este objetivo se combinaron técnicas de investigación cualitativas y cuantitativas, aplicadas a académicos y directivos de las instituciones afiliadas a la Asociación Nacional de Facultades y Escuelas de Contaduría y Administración (ANFECA). Los resultados sugieren que la creatividad, la incorporación de la tecnología y el trabajo colegiado son elementos esenciales para promover la innovación educativa en las disciplinas contables y administrativas en México.
\end{abstract}

Palabras clave: innovación educativa, contaduría y administración, actualización docente. 


\title{
Perfil e práticas educativas do docente orientado à inovação nas escolas de negócios em México
}

\section{Resumo}

Para as escolas de negócios do México é particularmente transcendente responder às demandas sociais e de qualidade acadêmica do ensino superior. Perante o desafio das mudanças, o professor é o ator central nos processos de inovação educativa. O objetivo principal deste trabalho de pesquisa exploratória e descritiva foi conhecer as características do perfil e das práticas educativas de um professor inovador nas escolas mexicanas de negócios. Para alcançar este objetivo foram combinadas técnicas qualitativas e quantitativas de pesquisa, aplicadas a acadêmicos e diretores das instituições reunidas na Associação Nacional de Faculdades e Escolas de Contadoria e Administração (ANFECA). Os resultados sugerem que a criatividade, a incorporação da tecnologia e o trabalho associado são elementos essenciais para promover a inovação educativa nas disciplinas contáveis e administrativas em México.

Palavras chave: inovação educativa, contadoria e administração, atualização docente.

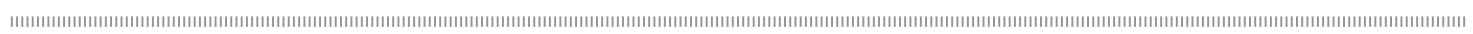

\section{Educational profile and practices of teachers oriented towards innovation in business schools in Mexico}

\begin{abstract}
It is of especial transcendence for business schools in Mexico to confront the demands for social and academic quality in higher education. Challenged by change, the teacher is the central actor in the processes of educational innovation. The main objective of this exploratory-descriptive piece of research was to get to know the characteristics of the profile and educational practices of an innovating teacher in business schools in Mexico. In order to achieve this, qualitative and quantitative research techniques were combined, applied to teachers and directors of institutions affiliated to the National Association of Faculties and Schools of Accounting and Management (ANFECA). Results suggest that creativity, the incorporation of technology and collegiate work are key elements for promoting educational innovation in accounting and management disciplines in Mexico.
\end{abstract}

Key words: educational innovation, accounting and management, teacher professional development.

Recepción: 4/8/10. Aprobación: 11/7/11. 


\section{Antecedentes. La innovación} educativa en las instituciones de

\section{educación superior}

El desarrollo social, los avances tecnológicos y la creciente globalización requieren de transformaciones claves en las instituciones de educación superior (IES). Algunos aspectos críticos en este proceso de transformación, particularmente para las IES mexicanas, son la integración de un sistema más orientado a los estudiantes, que incorpore la movilidad interinstitucional de los mismos, la puesta en práctica de nuevas estrategias didácticas y el desarrollo de formas complementarias de educación en las que se haga un uso efectivo de las nuevas tecnologías de información y comunicación (TIC) (Ibarra, 2002).

Ante estos desafíos, es necesaria la incorporación de una cultura de innovación en las IES. De acuerdo con Blanco y Messina (2000), la innovación educativa se define como un proceso encaminado a la solución de problemas de calidad, cobertura, eficiencia y efectividad en el ámbito educativo, resultado de un proceso participativo de planificación, que surge desde la práctica educativa del profesorado y que confronta las creencias de docentes y administrativos, y plantea formas alternativas de enseñar, aprender y gestionar.

Las innovaciones se caracterizan por una diversidad de formas, modalidades y alcances e implican tanto cambios en las actividades como en las actitudes. Los principales obstáculos a la innovación educativa son la centralización excesiva, la posición defensiva del personal docente, la ausencia de un agente de cambio, el enlace insuficiente entre teoría y práctica, y la falta de conocimientos acerca de los procesos educativos por parte de padres, funcionarios locales y administrativos del campo de la educación.

Con respecto a los aspectos mencionados, la Asociación Nacional de Universidades e Instituciones de Educación Superior (ANUIES, 2003) ha definido cinco ámbitos de innovación: 1) planes y programas de estudio, 2) proceso educativo, 3) uso de tecnologías de información y comunicación, 4) modalidades alternativas para el aprendizaje y 5) gobierno, dirección y gestión (véase tabla 1).

La educación superior y la investigación son componentes claves en el desarrollo de conocimientos, habilidades y actitudes que la sociedad demanda. Esto permite que los diferentes actores sociales puedan contar con las herramientas necesarias para tratar de erradicar la pobreza, lograr un desarrollo sustentable y un progreso en el alcance de las metas consensuadas por la comunidad internacional (Conferencia Mundial de Educación Superior, 2009). Dado el importante papel de las IES y la complejidad de los desafíos presentes y futuros, estas instituciones tienen la responsabilidad de mejorar la comprensión de cuestiones que presentan múltiples aristas, desde una dimensión social, económica, científica y cultural, lo que resalta la necesidad de que incrementen su mirada interdisciplinaria y promuevan el pensamiento crítico y la ciudadanía activa.

La educación superior, al tener un impacto en el desarrollo de la sociedad, es responsabilidad de diversos grupos de interés. Por ello acoge las expectativas de diferentes actores sociales sobre la formación de personas como agentes de cambio, y se espera que favorezca modelos e iniciativas que brinden una mayor equidad en las oportunidades de movilidad, promoción escolar y formación laboral. Las universidades en México deben avanzar hacia la internacionalización y modernización, con el fin de ampliar los horizontes académicos de su cuerpo docente en bien de sus estudiantes (Grediaga, 2006). Este cambio en la enseñanza debe estar enfocado en la formación de competencias acorde a la reestructuración de las sociedades contemporáneas occidentales (Moreno, 2009). 


\section{Tabla 1. Aspectos, objetivos e indicadores en relación con la innovación} educativa en las IES

\begin{tabular}{|c|c|c|}
\hline Aspecto & Objetivos de innovación & Indicadores de evaluación \\
\hline Planes y programas & Formación integral del estudiante & $\begin{array}{l}\text { - Fundamentación diagnóstica de calidad } \\
\text { - Criterios orientadores para la innovación } \\
\text { - Tutorías } \\
\text { - Nuevos modelos de evaluación, acreditación } \\
\text { y certificación por competencias } \\
\text { - Nuevos modelos de aprendizaje a través de } \\
\text { nuevas tecnologías }\end{array}$ \\
\hline \multirow{4}{*}{ Proceso educativo } & Proceso de aprendizaje & $\begin{array}{l}\text { - Grado de aprendizaje significativo, } \\
\text { autogestionado, metacognitivo e integral }\end{array}$ \\
\hline & Proceso de enseñanza & $\begin{array}{l}\text { - Grado de acompañamiento, mediación } \\
\text { y coparticipación en la construcción del } \\
\text { conocimiento }\end{array}$ \\
\hline & Formación docente & - Grado de formación para la innovación \\
\hline & $\begin{array}{l}\text { Recursos y materiales del } \\
\text { aprendizaje }\end{array}$ & $\begin{array}{l}\text { - Grado de diseño y elaboración de materiales } \\
\text { y medios didácticos y la generación de } \\
\text { modelos de diseño flexibles }\end{array}$ \\
\hline Uso de TIC & Apoyo en el proceso de aprendizaje & $\begin{array}{l}\text { - Grado de uso de las TIC como herramientas } \\
\text { de apoyo al proceso de aprendizaje }\end{array}$ \\
\hline $\begin{array}{l}\text { Modalidades } \\
\text { alternativas para el } \\
\text { aprendizaje }\end{array}$ & $\begin{array}{l}\text { Modelo alternativo con un } \\
\text { currículo flexible, con movilidad y } \\
\text { diversificación, atendiendo a las } \\
\text { necesidades de los estudiantes }\end{array}$ & $\begin{array}{l}\text { - Grado de flexibilidad del curricula } \\
\text { - Porcentaje de alumnos que participan en } \\
\text { programas de movilidad } \\
\text { - Becas destinadas a programas de movilidad. } \\
\text { - Técnicas y medios para el autoaprendizaje }\end{array}$ \\
\hline \multirow{3}{*}{$\begin{array}{l}\text { Gobierno, dirección y } \\
\text { gestión }\end{array}$} & $\begin{array}{l}\text { Flexibilización de la legislación } \\
\text { educativa }\end{array}$ & $\begin{array}{l}\text { - Grado de flexibilidad de la legislación } \\
\text { educativa hacia la innovación }\end{array}$ \\
\hline & $\begin{array}{l}\text { Conocimiento sobre otras } \\
\text { experiencias }\end{array}$ & $\begin{array}{l}\text { Número de investigaciones desarrolladas } \\
\text { orientadas al estudio de la innovación } \\
\text { educativa }\end{array}$ \\
\hline & Capacitación del personal & - Programas de capacitación de profesores \\
\hline
\end{tabular}

Fuente: elaboración propia basado en ANUIES (2003). 
Las innovaciones pueden definirse como procesos de resolución de problemas, y por tanto una innovación educativa efectiva es aquella que logra integrarse con otros componentes del proceso educativo o pedagógico, con los cuales necesariamente interactúa y se complementa para lograr frentes más amplios de mejoramiento de la calidad, de la cobertura y de la eficiencia (Blanco y Messina, 2000). La innovación en el sistema educativo, sin embargo, no es un proceso sencillo en cuanto puede encontrar una posición defensiva en el personal docente, en los padres y en las autoridades educativas. Así, se considera que el proceso de innovación en el sistema educativo tiene más alcance cuando surge del mismo profesorado, y es éste, por ende, quien cuestiona y plantea otra forma de enseñar y aprender.

Para avanzar en este proceso de mejora en la educación superior, se hace necesario el desarrollo y fortalecimiento de competencias para la innovación en el profesorado. Rueda y Luna (2008) señalan que la Red de Investigadores sobre la Evaluación de la Docencia (RIED), por medio de diversos trabajos de investigación, ha reafirmado que la docencia universitaria es uno de los agentes centrales del desarrollo educativo y que la evaluación de su práctica con un enfoque formativo y de perfeccionamiento, es la vía de acceso para su mejora permanente.

Según Saldívar (2007), la práctica docente implica reconocimiento, adaptación, actualización e innovación; este autor reconoce la importancia de que el docente sea un aprendiz permanente y así pueda regenerar y evolucionar su método y proceso de enseñanza. El docente debe ser capaz y estar dispuesto a aprender y utilizar recursos educativos diversos con la finalidad de convertir el proceso de enseñanza-aprendizaje en un proceso más interactivo y llamativo para todos los actores involucrados. Por ello, es de vital importancia, como lo afirma Saldívar, que la práctica docente se adapte a los requerimientos sociales, económicos y políticos que demanda la sociedad y el entorno en general.

La importancia del docente en el cambio educativo no tiene discusión, sin embargo Díaz Barriga (2005) afirma que la política educativa actual dificulta el papel del docente en cuanto a que algunas demandas o requerimientos no convergen entre sí. A veces se le pide un rol erudito, en otras un rol formativo en el desarrollo de habilidades, o bien fortalecer el dominio de la información; en otros casos, la obtención de conocimiento ordenado en una estructura disciplinaria, mientras que en otros se promueve la idea de la participación, del desarrollo de un proceso (Díaz Barriga, 2005). Ante esta carga o andamiaje de requisitos, un cuestionamiento significativo sería si se le ha capacitado para cambiar e innovar sobre su propia práctica docente.

Estudios recientes como los de Almerich y otros (2005), Madden y otros (2005) y Mahdizadeh y otros (2008), coinciden en señalar la necesidad de formación de competencias orientadas a la innovación en los docentes, como un medio de cambiar sus actitudes hacia los procesos de cambio y mejora en las instituciones.

\section{El reto de la innovación educativa en las escuelas de negocios en México}

En virtud de las demandas educativas requeridas por la sociedad, las facultades y escuelas de negocios a nivel mundial están replanteando y rediseñando sus programas. En estas escuelas ha surgido una corriente de educación progresista que no sólo critica el proceso de enseñanza-aprendizaje tradicional, sino que además propone una concepción educativa más amplia diseñada para facilitar la educación para la vida y para el ejercicio de la ciudadanía, mediante la formación de un pensamiento crítico (Zaid, 2002). El objetivo, como ya se ha mencionado, es preparar a los 
educandos con las herramientas necesarias para hacer frente a las nuevas estructuras económicas y sociales.

La innovación educativa en México es ahora un requerimiento que demanda la asociación de diferentes entes que permita la formulación de un plan académico consensuado en los diferentes niveles educativos. En los estudios superiores de contabilidad y administración, la Asociación Nacional de Facultades y Escuelas de Contaduría y Administración (ANFECA) es la entidad que vela por el mejoramiento y actualización de los planes y programas de estudio, así como por el mejoramiento académico del profesorado. La importancia de la ANFECA, fundada en 1949, radica no sólo en su misión de mejorar la educación sino en que agrupa a cerca de 247 instituciones en todo el país (ANFECA, 2010), por lo cual cuenta con una visión nacional consensuada.

La innovación educativa en las escuelas de negocios está integrada por los cambios y mejoras en la docencia y en la investigación, por la actualización docente y profesional y por la adecuación y adaptación al entorno actual. Además, la innovación educativa implica que exista concordancia entre los planteamientos y necesidades que demanda la sociedad, así como solucionar las necesidades que enfrentan las instituciones de educación superior en la actualidad y la generación de capital intelectual.

La sociedad constantemente demanda un cambio en la educación que permita y continúe estimulando su desarrollo. Por ello, es importante que las autoridades gubernamentales e instituciones sociales y educativas colaboren para llevar a cabo con éxito esta innovación que la sociedad demanda en la educación. Algunas acciones que se deben de realizar para implementarla son la elaboración de diagnósticos institucionales, la actualización del docente, la comunicación e interacción social, la actualización permanente de planes y programas de estudio, entre otros. Sin embargo, algunos de los factores que dificultan la innovación educativa son el número de alumnos en el aula, el perfil del estudiante, la actitud del profesorado hacia la innovación, la identidad institucional, el enfoque educativo, la falta de recursos financieros y humanos, el uso inadecuado de la tecnología y la falta de trabajo colaborativo académico (ANFECA, 2007, 2009). Es necesario profundizar más en el contexto de las escuelas de negocios en México, para comprender los procesos de innovación educativa que les son pertinentes, enfatizando el papel del profesorado.

\section{Metodología de investigación y contexto}

La actualización del docente constituye un punto central para lograr promover una educación superior de calidad en los niveles nacional e internacional. Con el propósito de comprender mejor la relación entre las prácticas docentes y la innovación educativa, se definió como objetivo general de investigación el siguiente: describir las características del perfil y de las prácticas docentes en los profesores universitarios con orientación a la innovación educativa en las escuelas de negocios en México. Como objetivos particulares se definieron los siguientes: 1) conocer los elementos de las prácticas educativas innovadoras, y 2) determinar las necesidades de actualización y capacitación de los profesores para favorecer la innovación educativa en las escuelas de negocios en México.

La pregunta de investigación que sustenta este estudio fue la siguiente: ¿Cuáles son las características del perfil y de las prácticas docentes de los profesores universitarios orientados a la innovación educativa en las escuelas de negocios en México?

Para lograr el propósito del estudio y dar respuesta a la pregunta de investigación planteada, se llevó a cabo una investigación con alcance exploratorio-descriptivo y de enfoque mixto. Se 
utilizaron técnicas de investigación cualitativas como entrevistas semiestructuradas, grupos de discusión y foros de discusión virtual, y cuantitativas, mediante la aplicación de cuestionarios, para la obtención de datos, que además permitieron la triangulación entre los mismos, a fin de dar robustez al análisis de los resultados, conclusiones y recomendaciones emitidas.

Las técnicas de investigación cualitativas permitieron la identificación de categorías e indicadores relacionados con la innovación educativa, consistentes con conceptos previos como la incorporación de tecnologías de información, las prácticas colegiadas y la vinculación. Estas categorías fueron posteriormente incorporadas en los cuestionarios. En el aspecto cuantitativo, se aplicaron técnicas de estadística descriptiva con el fin de jerarquizar las categorías e indicadores sujetos a estudio.

El contexto de la investigación estuvo representado por dos unidades de análisis: la primera representada por los cuerpos académicos de las facultades y escuelas de negocios afiliadas a ANFECA, y la segunda por los miembros del Consejo Nacional Directivo de esta asociación en México. Dentro del primer grupo también se consideró a secretarios académicos, coordinadores de carrera, presidentes de academia y a los docentes como los informantes clave, puesto que de manera directa tienen relación con las temáticas centrales de análisis: el perfil del docente y sus prácticas educativas. El segundo grupo estuvo integrado por directores de escuela, de facultad o bien de programa académico; este grupo aportó la perspectiva institucional al estudio.

El estudio se llevó a cabo durante el periodo enero-mayo de 2010. En la resolución de los cuestionarios institucionales participaron 58 instituciones afiliadas de un total de 276; en los foros y grupos de discusión participaron alrededor de 85 académicos, y en las entrevistas semiestructuradas participaron 12 directores de escuelas o facultades, con representación de las siete zonas de todo el país. Lo anterior se llevó a cabo con el propósito de obtener información relacionada con las actividades de formación docente orientada a la innovación en las facultades y escuelas de negocios, así como para la identificación de aspectos relacionados con las cualidades de sus docentes y sus prácticas educativas.

Aunque la metodología amplia descrita en este apartado permitió la obtención de datos referentes a los procesos de innovación en las escuelas de negocios en México, a continuación se presentan, para efectos de este reporte de investigación, los resultados obtenidos en los aspectos particulares de perfil docente y prácticas innovadoras.

\section{Discusión de los Resultados En relación con el perfil docente orientado a la innovación educativa}

En el nivel institucional, con respecto a las habilidades, conocimientos y actitudes del perfil docente que se consideran necesarias para promover el proceso de innovación educativa, los resultados obtenidos en orden de importancia son: capacidad de aplicar los conocimientos; conocimiento sobre el área de estudio; compromiso ético; capacidad de abstracción, análisis y síntesis; capacidad de aprender y actualizarse permanentemente; capacidad para identificar, planear y resolver problemas; habilidades en el uso de las tecnologías de información y comunicación; capacidad para tomar decisiones; valoración y respeto por la diversidad y la multiculturalidad, y finalmente, la capacidad de investigación.

Por otra parte, en el nivel del profesorado, los principales conocimientos, habilidades y actitudes relacionadas con la innovación, en orden de importancia de mayor a menor, son: manejo de TIC; creatividad; liderazgo; adaptabilidad; espíritu emprendedor; comunicación efectiva, y capacidad de motivar. 
Los resultados obtenidos por parte del profesorado, sugieren un mayor énfasis en el desarrollo de competencias relacionadas con la comunicación y las relaciones interpersonales, y un menor énfasis en los conocimientos técnicos. Los resultados, tanto en el nivel institucional como en el nivel del profesorado, confirman la importancia que tienen en la actualidad las TIC en las áreas laboral y académica. Como conclusión, las TIC son muy importantes para construir la plataforma de la innovación educativa en las instituciones de educación superior.

Directivos y docentes coinciden en que el aprendizaje continuo, el liderazgo, la creatividad, el manejo de TIC y la adaptabilidad son las principales cualidades requeridas para la innovación educativa. Un aspecto a resaltar de los resultados de esta investigación es que los docentes consideraron a la actitud emprendedora y la comunicación efectiva dos competencias muy importantes, en cambio los directivos otorgaron a estas competencias un menor grado de importancia. Los directivos destacan que el aprendizaje continuo y el liderazgo son las competencias que más deben desarrollarse en los docentes orientados a la innovación.

\section{En relación con las características de las prácticas educativas innovadoras}

Además del desarrollo y fortalecimiento de un perfil docente orientado a la innovación, es muy importante la construcción de prácticas educativas orientadas hacia la innovación educativa. Los resultados obtenidos en esta investigación indican que las autoridades y actores encargados de administrar la educación deben procurar que las prácticas educativas actuales se apoyen en las nuevas tecnologías de información y comunicación, creen vínculos con otras instituciones educativas y empresariales, fomenten el aprendizaje y exposición de los alumnos en diferentes idiomas y contextos culturalmente diversos, utilicen materiales didácticos actualizados, permitan la integración del conocimiento e incorporen la dimensión ético-social en los contenidos curriculares.

De la información recopilada con las entrevistas se puede observar que los catedráticos asignan un alto grado de importancia al uso de las TIC y a la vinculación con las organizaciones empresariales y sociales para llevar a cabo la innovación educativa. También se puede identificar que los catedráticos dan significancia a la implementación de una metodología dinámica de enseñanza, a un aprendizaje basado en casos de estudios y trabajos de investigación, y a la creación y utilización de redes académicas, si se quiere lograr con éxito la innovación en la educación superior. Esta idea es apoyada por los directivos, quienes afirmaron además que no es muy frecuente la vinculación de lo estudiado con las necesidades sociales y empresariales ni la utilización de redes académicas, reconociendo por tanto que la labor de autoridades educativas en la actualidad debe procurar facilitar las condiciones para que esta interacción suceda.

Los resultados obtenidos en este estudio exploratorio-descriptivo, si bien confirman los aspectos esenciales asociados con la innovación educativa (Blanco y Messina, 2000; Ibarra, 2002; ANUIES, 2003), en tanto al proceso de solución de problemas, la incorporación de tecnologías de información y la participación del profesorado, aportan una perspectiva desde las escuelas de negocios en México y permiten ampliar el diálogo sobre la innovación en estas disciplinas.

Es necesario ampliar la muestra de profesores y directivos participantes con el fin de poder obtener una mayor cantidad de datos que permitan establecer relaciones entre las categorías e indicadores y otras variables, por ejemplo el tamaño de la institución, el financiamiento de la misma (público o privado) y la región geográfica, lo cual permitirá una interpretación más amplia del fenómeno de la innovación educativa en las escuelas de negocios. 


\section{Conclusiones: hacia un docente innovador en las escuelas de negocios}

La educación es un factor determinante para mejorar la calidad de vida en las personas, por lo que los docentes se convierten en la mejor alternativa para formar personas profesionales bien preparadas, en beneficio de la sociedad. Las cualidades consideradas como innovadoras para el docente son apropiadas para mejorar el proceso de enseñanza-aprendizaje, considerando los requerimientos actuales de la sociedad. Se plantea la posibilidad de que ANFECA sea el foro óptimo para discutir sobre el perfil del docente orientado a la innovación, dada su característica de ser un espacio de carácter nacional donde convergen representaciones de las IES públicas y privadas.

En relación con el análisis de los resultados obtenidos en este estudio, el perfil del docente orientado a la innovación en las escuelas de negocios, posee las destrezas y capacidades siguientes:

- Uso efectivo de la tecnología en el proceso enseñanza-aprendizaje

- Trabajo colegiado

- Administración del proceso de enseñanza -aprendizaje

- Enfoqueéticodelprocesoenseñanza-aprendizaje

- Innovación educativa en el aprendizaje activo

- Actualización profesional y disciplinaria con enfoque nacional e internacional

- Comunicación efectiva

- Pensamiento crítico con orientación hacia la creación y divulgación del conocimiento

Sin embargo, aunque estas cualidades son reconocidas por académicos y directivos de las instituciones afiliadas como esenciales en los procesos de innovación educativa, son también las que se reconocen con menor presencia dentro de los perfiles actuales de los profesores en las instituciones afiliadas.
Es necesario proponer, discutir y analizar estas características, para definir con mayor claridad el perfil del docente orientado a la innovación educativa en las instituciones afiliadas, además de incorporar en el nivel institucional criterios de evaluación del desempeño orientados a incentivar el desarrollo de profesores innovadores.

En complemento al perfil de un profesor orientado a la innovación y como resultado del análisis efectuado en este trabajo sobre las opiniones de los académicos y directivos de las instituciones afiliadas, las prácticas educativas innovadoras en las escuelas de negocios poseen las características siguientes:

- Ponen en práctica destrezas docentes orientadas a la innovación en el proceso de enseñanza-aprendizaje, como el pensamiento crítico y la reflexión.

- Son compartidas mediante redes académicas para intercambiar experiencias educativas.

- Incorporan las TIC en el proceso de enseñanza-aprendizaje.

- Sus contenidos disciplinares corresponden a planes de estudio actualizados y enfocados al desarrollo de conocimientos, actitudes, habilidades y valores.

- Están vinculadas con el sector profesional, el gobierno y las empresas.

Con la finalidad de lograr prácticas educativas innovadoras, es necesario, entonces, desarrollar la capacidad de innovación de los profesores, mediante programas de capacitación y actualización. Además, estos programas de capacitación deben procurar facultar a los profesores para que puedan incorporar las nuevas TIC en sus actividades de enseñanza orientadas al desarro1lo de competencias en los estudiantes. Es muy importante que estas prácticas educativas innovadoras implementadas por los profesores sean 
documentadas y compartidas, con el propósito de crear un acervo de prácticas educativas en beneficio de la comunidad académica y empresarial.

Con base en la información recopilada, se puede afirmar que la innovación educativa requiere de los siguientes elementos: tecnología, metodologías de enseñanza-aprendizaje novedosas y actualización de las competencias pedagógicas y disciplinares de los académicos. También permite afirmar que el aprendizaje es significativo cuando el maestro logra incluir en el proceso de enseñanza-aprendizaje problemas reales, y establecer un vínculo con los diferentes sectores de la sociedad. Es indiscutible que la apertura al cambio es el factor fundamental para llevar a cabo procesos educativos innovadores.

\section{Referencias}

Almerich, G., J. M. Suárez, N. Orellana, C. Belloch, R. Bo e I. Gastaldo (2005), "Diferencias en los conocimientos de los recursos tecnológicos en profesores a partir del género, edad y tipo de centro", en RELIEVE, vol. 11, núm. 2, pp. 127-146.

ANFECA (2010), "Objetivos estratégicos", México, ANFECA, < <http://www.anfeca.unam.mx> [consulta: mayo 2010].

ANFECA (2009), "Modelos pedagógicos en las escuelas de negocios", Ponencia central, L Asamblea Nacional, México, ANFECA, disponible en $<$ http://www.anfeca.unam.mx $>$.

ANFECA (2007), "Innovación educativa en las escuelas de negocios", Ponencia central, XLVIII Asamblea Nacional ANFECA, México, ANFECA, disponible en $<\mathrm{http}$ // $/$ www.anfeca.unam.mx $>$.

ANUIES (2003), Documento estratégico para la innovación en la educación superior, México, ANUIES.

Blanco R. y G. Messina (2000), "Estado del arte sobre las innovaciones educativas en América Latina", Colombia, Convenio Andrés Bello, UNESCO.
Este trabajo permite también afirmar la necesidad de evolucionar pedagógica y profesionalmente con el fin de dar respuesta a las demandas de los grupos de interés, tanto internos como externos, y así poder incentivar, en forma permanente, la creatividad e innovación en la práctica académica. Ante el desafío de mejorar la calidad y la pertinencia de la educación en el ámbito de negocios en México, es necesario desarrollar y compartir el talento académico de los profesores, ya que el profesor es el actor principal del proceso de cambio e innovación educativa. Por ello, la estructura, la tecnología y las políticas institucionales se hacen efectivas solamente en las manos del profesor que las utiliza, las promueve y las vive junto con sus alumnos, por medio de prácticas educativas innovadoras.

Conferencia Mundial de Educación Superior (2009), "Las nuevas dinámicas de la educación superior y de la investigación para el cambio social y el desarrollo", en Revista de la Educación Superior, vol. 31, México, ANUIES [consulta: mayo 2010].

Díaz-Barriga, A. (2005), "El profesor de educación superior frente a las demandas de los nuevos debates educativos", en Perfiles Educativos, vol. 27, núm. 108, México, CESU-UNAM.

Grediaga, R. (2006), "La internalización de las universidades en México", en Revista de la Educación Superior, enero-marzo, núm. 137, México, ANUIES.

Ibarra Colado, Eduardo (2002), "La nueva universidad en México: transformaciones recientes y perspectivas", en Revista Mexicana de Investigación Educativa, vol. 7, núm. 14, México, Consejo Mexicano de Investigación Educativa, <http://www.comie.org. $\mathrm{mx} / \mathrm{vl} /$ revista/portal.php $>$ [consulta: abril 2010].

Madden, Ford, Miller y Levy (2005), "Using the Internet in teaching: the views of practitioners (A survey of the views of secondary school teachers 
in Sheffield, UK), en British Journal of Educational Technology, vol. 36, núm. 2, pp. 255-280.

Mahdizadeh, Biemans y Mulder (2008), "Determining factors of the use of e-learning environments by university teachers", en Computers \& Education, núm. 51, pp. 142-154.

Moreno, T. (2009), "Competencias en educación superior: un alto en el camino para revisar la ruta de viaje", en Perfiles Educativos, vol. 31, núm. 124, México, IISUE-UNAM, <http://132.248.192.201/ seccion/perfiles/2009/n124a2009/mx.peredu.2009. n124.p69-92.pdf $>$ [consulta: abril 2010].

Rueda, M. y E. Luna (2008), "La docencia universitaria y su evaluación", en Revista Electrónica de Investigación Educativa, núm. especial, México, Universidad Autónoma de Baja California, < http://redie.

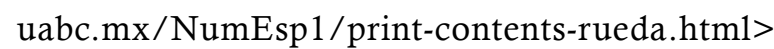
[consulta: 12 abril 2010].

Saldívar, H. (2007), "La globalización y su impacto en las políticas de educación superior", Editorial Plaza $y$ Valdés, México.

Zaid, M. (2002), "Spinning disciplines: critical management studies in the context of the transformation of management education", en Organization, <http:// org.sagepub.com/content/9/3/365.abstract $>$ [consulta: 15 abril 2010].

\section{Cómo citar este artículo:}

Lavín-Verástegui, Jesús y Gabriela-María Farías-Martínez (2011), "Perfil y prácticas educativas del docente orientado a la innovación en las escuelas de negocios en México", en Revista Iberoamericana de Educación Superior (RIES), México, UNAM-IISUE/Universia, Vol. III, núm. 6, pp. 117-127, http://ries.universia.net/index.php/ries/article/ view/147 [consulta: fecha de última consulta]. 